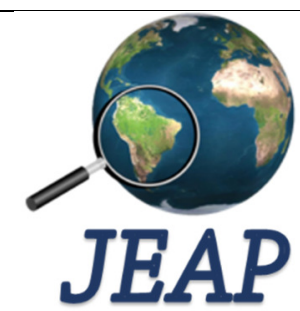

ISSN: 2525-815X

\section{Journal of Environmental} Analysis and Progress

Journal homepage: www.jeap.ufrpe.br/ http://dx.doi.org/10.24221/jeap.2.3.2017.1473.341-348

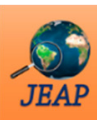

Journal of Environmental Analysis and Progress

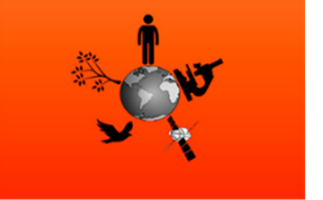

\title{
Análise conceitual do Building Information Modeling-BIM e City Information Modeling-CIM e contribuições na construção das cidades sustentáveis
}

\section{Conceptual analysis of Building Information Modeling-BIM and City Information Modeling-CIM and contributions in the construction of sustainable cities}

Jadson Freire da Silva ${ }^{\mathrm{a}}$, Rutt Keles Alexandre da Silva ${ }^{\mathrm{a}}$, Marcelo Olímpio dos Santos ${ }^{\mathrm{b}}$, Mariana Gonçalves Pires Lopes ${ }^{\mathrm{c}}$, Isabela de Oliveira Barros ${ }^{\mathrm{d}}$, Elvis Bergue Mariz Moreira ${ }^{\mathrm{e}}$, Henrique dos Santos Ferreira ${ }^{\mathrm{f}}$

${ }^{a}$ Universidade Federal de Pernambuco-UFPE, Programa de Pós-Graduação em Desenvolvimento e Meio AmbientePRODEMA. Departamento de Ciências Geográficas, Cidade Universitária, Recife, Pernambuco, Brasil. CEP: 50740540. E-mail oijadsonfreire@icloud.com, ruttgeografia@gmail.com.

b Universidade Federal de Pernambuco-UFPE, Programa de Pós-graduação em Desenvolvimento Urbano-MDU. Departamento de Arquitetura e Urbanismo, Avenida da Arquitetura, s/n, Universidade Federal de Pernambuco, Cidade Universitária, Recife-PE-Brasil. CEP: 50740-550.

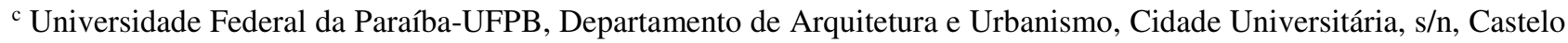
Branco III, João Pessoa-PB. CEP: 58051-900.

d Universidade Federal de Pernambuco-UFPE, Departamento de Ciências Geográficas, Cidade Universitária, Recife-PE, Brasil. CEP: 50740-540. E-mail isa.oliver20@gmail.com.

e Universidade Federal do Oeste da Bahia-UFOB, Departamento de Geografia. Rua Professor José Seabra de Lemos, n. 316, Recanto dos Pássaros, Barreiras-BA. CEP: 47808-021. E-mail: elvisberg@ gmail.com.

${ }^{\mathrm{f}}$ Universidade Federal de Pernambuco-UFPE, Programa de Pós-Graduação em Geografia-PPGEO, Departamento de Ciências Geográficas, Cidade Universitária, Recife-PE, Brasil. CEP: 50740-540. E-mail: henriquehs86@hotmail.com.

A R T I CLE I N F O

Recebido 02 Jul 2017

Aceito 29 Jul 2017

Publicado 31 Jul 2017

\begin{abstract}
A B S T R A C T
The rapid development of the environment and urban planning are increasingly demanding of management, celerity, and readiness in solving difficulties. Considering the development in the environment, companies invest in technologies and information modelling; when the State administration adopts these, it can contribute to the improvement of the operation of several aspects, reflecting the social and environmental quality of the regions of interest. Taking into account The rapid dissemination of communication and the advancement of technology, this work aimed to analyze the concepts that approach the considerations of Building Information Modelling-BIM and City Information Modeling-CIM for cities, presenting their functionalities and benefits. The methodological procedures that conditioned the organization of the data presented here were fundamentally a bibliographical survey to select the literature that would compose the theoretical basis of this research. The conception of BIM and its benefits to the academic community, the revolutionary arrival of CIM and the contribution of these forms of modelling to the formation of sustainable cities are analyzed and discussed. It is concluded that the modelling of BIM and CIM and its vast and integrated database can provide several benefits to the design, engineering, architecture and related areas, assisting in the management of projects, observing the area available for construction and the natural resources of the region. It is possible to create cities in the sustainable model, potentializing energy generation, water and soil use, population transition/seasonality and reducing unnecessary costs.
\end{abstract}


Keywords: Environment, territorial management, technology.

\begin{abstract}
R E S U M O
O rápido desenvolvimento do meio ambiente e planejamento urbanos exige mais da gestão, como rapidez e facilidade na solução de percalços. É nesse ambiente de desenvolvimento que as empresas investem em tecnologias e na modelagem de informações; quando estas são adotadas pela administração de estados pode contribuir com a melhoria do funcionamento de vários fluxos, refletindo na qualidade social e ambiental das regiões de interesse. Observando a rápida disseminação da comunicação e avanço da tecnologia, este estudo objetivou analisar os conceitos sobre as considerações do Building Information Modeling-BIM e City Information Modeling- CIM para as cidades, apresentando suas funcionalidades e benefícios. Os procedimentos metodológicos condicionantes à organização dos dados constituíramse de levantamento bibliográfico para seleção da literatura, para compor a base teórica desta investigação. A conceituação do BIM e seus benefícios para a comunidade acadêmica, a chegada revolucionária do CIM e a contribuição destas formas de modelagem para a formação das cidades sustentáveis são analisadas e discutidas. Conclui-se que a modelagem oriunda do BIM e CIM e seu vasto e integrado banco de dados pode proporcionar diversos benefícios para os ramos do design, engenharia, arquitetura e áreas afins, auxiliando na gestão de projetos, observando a área disponível para construção e os recursos naturais da região, podese criar cidades no modelo sustentável, potencializando a geração de energia, uso da água e solo, transição/sazonalidade populacional e reduzindo custos desnecessários. Palavras-Chave: Meio Ambiente, gestão territorial, tecnologia.
\end{abstract}

\section{Introdução}

O rápido desenvolvimento que ocorre nas áreas de meio ambiente e planejamento urbano está exigindo cada vez mais da gestão, a rapidez e a facilidade na solução de percalços. É nesse ambiente de desenvolvimento que as empresas investem em tecnologias e processos que consigam alinhar soluções habituais das grandes cidades e a agilidade exigida, tendo como propósito o aumento de rentabilidade e satisfação do cliente. Essas evidências beneficiam o Building Information Modeling-BIM, modelo de informação que aumenta a produtividade e reduz erros e custos (Aranda et al., 2008). A integração da tecnologia, processos e pessoas é essencial para a organização e ordem desse sistema, que foi adotado por diversas entidades e indústrias reguladoras de vários países, tais como o Instituto Americano de Arquitetos-AIA (EUA), a Administração geral de serviços-GSA (Grã-Bretanha) e a Instituição Britânica de Padrões-BSI (Inglaterra). Estas entidades junto a uma equipe multidisciplinar continuam a traçar caminhos e compartilhar conhecimentos acerca dos benefícios coletivos associados a abordagem do BIM recebendo suporte e atualizações.

Um dos conceitos que pode ser considerado inovador na área, baseia-se no próprio desenvolvimento do BIM e denomina-se City Information Modeling-CIM. O CIM é basicamente uma extensão do BIM aplicada a bairros ou cidades inteiras. Seu objetivo geral é possibilitar a integração de modelos elaborados em plataformas BIM, de modo a criar uma réplica digital da cidade, que poderá ser utilizada como base para a realização de análises e simulações sofisticadas. Assim como o BIM, o CIM pode ajudar na melhor integração de diferentes aspectos da cidade e, eventualmente, colaborar para uma melhor integração de diferentes estruturas e serviços dentro da cidade, permitindo que sua operação seja otimizada (PPGAU, 2015).

A modelagem de informações quando é adotada pelas administrações de estados, cidades ou distritos, pode contribuir com a melhoria do funcionamento de vários fluxos, assim, refletindo na qualidade social e ambiental das regiões de interesse, auxiliando no suporte a decisões da gestão. Assim, observando a rápida disseminação da comunicação, avanço da tecnologia e nascimento de novas formas para gestão de cidades, este estudo tem como objetivo uma análise dos conceitos que acercam as considerações do BIM e CIM para as cidades, apresentando suas funcionalidades e benefícios.

\section{Material e Métodos}

Os procedimentos metodológicos condicionantes à organização dos dados aqui apresentados, constituíram-se fundamentalmente de levantamento bibliográfico para seleção da literatura que viria a compor a base teórica desta pesquisa. Um argumento útil para a escolha deste tipo de análise é o fato da mesma condicionar uma visão geral sobre as narrativas que permeiam determinado assunto. Esta abordagem da investigação é, portanto, particularmente importante para a solução do problema central da pesquisa, haja vista a dispersão dos dados (Gil, 
2008) no meio tecno-científico-informacional (Santos, 1994). Neste caso, o acompanhamento das crescentes evoluções comuns às áreas da tecnologia se fazem necessárias.

As fontes de pesquisa consultadas durante o processo de planejamento e escrita do artigo são de origem nacional e internacional. Recorreu-se às plataformas do Google Acadêmico, CAPES e Scielo, tendo como premissa as palavraschave: BIM, CIM, Building Information Modeling e City Information Modeling; obtendo periódicos científicos, com significativo fator de impacto, visando adquirir uma fundamentação atualizada na busca sistemática pelos termos indexadores da investigação.

Ressalta-se a pertinência de pesquisas bibliográficas desta natureza na área do conhecimento científico que se volta à geotecnologia. Pois, são estudos como estes que auxiliam na reflexão sobre procedimentos aliados ao bem-estar social, mediante ponderação das alternativas que o meio tecnológico apresenta e os entraves que devem ser ultrapassados para a otimização numa aplicabilidade futura.

\section{Resultados}

Segundo Khemlani (2013), o Building Information Modeling-BIM é um modelo digital, representado por um prolífico banco de dados que auxilia na redução de tempo e custo da construção ou obra, sendo procurado e reforçado por um time que contribui, colabora e difunde as facilidades de gestão descobertas para a construção (Rezgui, Beach \& Rana, 2013). Os professores da universidade de Tesside-UK, Dawood \& Iqbal (2010) afirmam que esse modelo digital tem como foco principal o trabalho colaborativo com práticas organizacionais, tendo elevado valor para a indústria de construção, suportando consistentes e coordenados fluxos de informações para beneficiamento do ciclo da vida dos projetos urbanísticos.

A organização urbanística Alliance (2009) comenta que o BIM é um fenômeno de taxas crescentes e a indústria compreendeu os benefícios evidentes do sistema no que se refere à melhoria da qualidade de vida. Watson (2010) aponta que a origem da palavra veio de vendedores de softwares voltados aos designs de construções, com o objetivo direcionado a mostrar a essa comunidade científica a nova quebra de paradigmas que estava acontecendo no ramo dos softwares de design. Sah \& Cory (2009), bem como Gerber \& Rice (2010), o relacionam com o conhecimento de banco de dados, tecnologia e modelagem 3D junto com a colaboração multidisciplinar. Eastman et al. (2008) o define como uma modelagem tecnológica que é associada a uma série de processos para produção, comunicação e analise dos modelos de cidade, sendo relacionado com o princípio da interoperabilidade, isto é, a capacidade de um sistema de informação se comunicar com outro, semelhante ou não, de forma transparente.

O departamento de design e construção da cidade de Nova York-DDC/NY (2012) apresenta o Building Information Modeling como uma coleção digital de softwares e aplicativos voltados ao design para facilitação e coordenação de projetos. Podendo ser considerado como um processo que desenvolve documentações via plataforma virtual, construindo os grandes edifícios e criando as cidades planejadas antes destas serem construídas de fato. O departamento ressalta que o sistema é um modelo multidimensional, comportando as esferas 3D, 4D (tempo) e 5D (custo) e uma gama de informações que podem ser marcadas e anexadas para cada modelo como uma coleção de atributos.

Usando as ferramentas do BIM, os designs podem desenvolver diretamente em 3D (algo similar às linhas, blocos e arcos do auto CAD 2D), na medida em que o projeto é executado, toda quantidade de inteligência posta no trabalho é salva no banco de dados - database do BIM, podendo, posteriormente, usar esses dados para outros trabalhos.

\section{Benefícios do Building Information Modeling para a comunidade acadêmica}

Os benefícios do sistema BIM são múltiplos: a sua imediata visualização $3 \mathrm{D}$ contribuiu com a coordenação e organização dos processos (DDC/NY, 2012; Drogemuller, 2009). Ademais, Hamil (2013) enfatiza as funcionalidades de maximização a redução de resíduos durante a fase de design. A abordagem BIM é vital para a sustentabilidade econômica por dois motivos levantados por Osmani, Glass \& Prince (2006) e Oyedele et al. (2013), o primeiro é a diminuição dos custos de tempo e orçamento, os quais são essenciais para qualquer atividade civil e o segundo motivo é a clareza de projetos, visto que a má interpretação e as especificações são fontes fundamentais para os erros que ocasionam mais custos e tempo nas construções.

Diante disto, pode-se definir o sistema Building Information Model/Modeling como um software voltado aos engenheiros, designers, geógrafos e técnicos que estejam envolvidos com a construção civil ou a urbanização, onde o seu complexo banco de dados proporciona uma série de ferramentas que facilitam os processos de coordenação, comunicação, manutenção e análise de edifícios ou projetos urbanísticos. O reflexo disso é a migração atual das industrias do 
tradicional CAD para o BIM considerando sua eficiência e produção de informações.

A revolução do BIM: $O$ que é City Information Model/Modeling-CIM?

O desenvolvimento do Sistema de Informações Geográficas-GIS (Geographic Information System) trouxe para a comunidade um novo conceito de cidade digital. No entanto, segundo Stadler \& Kolbe (2007), os modelos GIS semanticamente são maus distribuídos. Os mesmos autores comentam que a plataforma BIM relaciona processos que envolvem a produção e a gestão de conteúdos digitais para representar características físicas de edifícios, sendo mais usado em projetos de baixas escalas. Assim, era necessário o desenvolvimento de um sistema que permitisse a modelagem de uma cidade como um todo, respeitando as atualizações dos softwares recentes, é nesse ambiente que nasce o City Information Model-CIM, um sistema que envolve as plataformas GIS e BIM para desenvolvimento de cidades (Amorim, 2015; Xu et al., 2014).

As definições de CIM são muito parecidas com às de BIM, sendo as vezes confundidas. Todavia, Gil, Almeida \& Duarte (2011) conceituam-no como um sistema baseado e um método de projeção urbano que integra as etapas de formulação, geração e avaliação de projetos urbanos apoiados sobre a plataforma CAD e GIS. Stojanovski (2013) revela que o CIM é a analogia do BIM em urbanismo, sendo um sistema urbano representado por símbolos em um espaço $2 \mathrm{D}$ e dentro de um espaço 3D. O sistema pode se estender ao uso do Sistema de Informações Geográficas-SIG no planejamento urbano como ferramenta de apoio, gestão e decisão (Webster, 1993). O CAD também pode constituir-se como ferramenta de apoio ao projeto (Dave \& Schmitt, 1994; Maguire, 2003).

Xu et al. (2014) definem o CIM como um banco de dados digital sendo utilizado pela engenharia civil facilitando a sua intercepção no sistema viário real, os softwares agregados ao CIM são interligados e permitem uma maior análise e desenvolvimento do design e da construção. $\mathrm{O}$ ambiente das cidades não é estático; várias dinâmicas devem ser consideradas, tais quais como pessoas, companhias empresariais e o fluxo de transportes, todos os citados são acatados como corpos ativos das cidades, e não apenas as edificações.

Considerando a estrutura do CIM, Xu et al. (2014) afirmam que as divisões básicas do software se desdobram em cinco partes/seções ou modelos: Building module ou seção de edificações; seção transportes; seção mobiliária; seção de corpos hídricos e a seção Mechanical, Eletrical and Plubing-MEP (mecânico, elétrico e canalização), onde se faz uso da tecnologia BIM para alimentar o modelo, e a tecnologia GIS para georreferenciar cada parte da cidade em estudo.

O módulo das edificações define-se pelas estruturas diversificadas dos prédios existentes numa grande cidade - onde se observa uma variação arquitetônica/estrutural temporal. Por estes motivos, a alimentação do sistema CIM demanda tempo e a busca de diferentes meios para digitalização de dados antigos. A segunda seção diz respeito aos transportes e faz referência às estradas; quanto às informações de custos, tamanho, tráfego e posicionamento de veículos, esta seção quando bem alimentada realiza o monitoramento em tempo real, cenários de tráfego e seu monitoramento.

No módulo mobiliário refere-se aos ambientes públicos da cidade, como os jardins, parques, praças e ambientes que proporcionam uma dinâmica de pessoas na cidade. Coletar informações sobre essas áreas é significativo, pois a gestão ao observar esta região pode potencializar a qualidade de vida para os residentes permanentes e temporários. A seção MEP retrata as conexões que as edificações na cidade necessitam: drenagem, elétrica, canalizações, estrutura mecânica, etc.

O módulo de corpos hídricos contém dados sobre os rios, lagos, oceanos, escoamento superficial, regime de chuvas, pluviometria, qualidade da água, entre outros. Esses dados auxiliarão a gestão com o controle da poluição, disponibilidade de água por região, monitoramento de áreas de riscos e na qualidade de ecossistemas inclusos na área de desenvolvimento.

Vale destacar que o conjunto de complexidade dos modelos BIM e CIM o colocam no patamar de 6 dimensões, sendo o BIM/CIM representando o 3D, o Schedules-Critical Path Method-COM (método do caminho crítico), algoritmo utilizado na confecção de projetos (4D), Cost Estimates - custos estimados (5D) e especificações para simular e validar (6D). Diante do exposto, pode-se afirmar que, para a construção de um sistema CIM, é necessário trabalho minucioso, pois muitas informações devem ser digitalizadas para serem inseridas nestes bancos de dados e isto exige tempo e mão de obra especializada. Além disso, alguns parâmetros digitalizados em CAD devem ser convertidos em outras plataformas, como por exemplo do CAD para o GIS, gerando novamente a exigência de mão de obra especializada. 


\section{Discussão}

A contribuição do BIM e CIM para a formação das cidades sustentáveis

Por intermédio da urbanização, fenômeno definido por Davis (1977) como o aumento do percentual da população urbana em relação à total, foi observado um aumento significativo, tanto no tamanho, quanto no número de cidades. No Brasil, por exemplo, a sazonalidade populacional ocorreu durante meados da década de 70 , onde as populações alocadas nas grandes cidades superaram a população rural, chegando, segundo dados apresentados pelo IBGE em seu censo demográfico (2010), a uma taxa de urbanização de $55,92 \%$ ainda naquela década. Informações do IBGE (2010), no seu último censo populacional, registraram que os dados populacionais corresponderam a cerca de 191 milhões de habitantes, dos quais 161 milhões viviam em zonas urbanas, implicando em um aumento intenso da população residente em zonas urbanas.

O crescimento acelerado das cidades, observado entre os anos 80 e 90 , foi desordenado e desprovido de planejamento estratégico, oriundo da inclusão de um contingente populacional superior ao que a cidade suporta. Essas regiões sofreram com atividades relacionadas à destruição de boa parte das condições ambientais e ao aparecimento de mazelas sociais (Sposito, 2004). É em função deste cenário de crescimento desordenado das grandes cidades que Rossetto (2003) reflete que, muitas vezes, o crescimento acelerado das cidades tem sido confundido com desenvolvimento e, no entanto, mesmo trazendo benefícios, nestes espaços existem impactos desastrosos para a qualidade de vida de seus habitantes e para as condições ambientais. Sendo assim, é de suma importância a existência de um planejamento para o desenvolvimento das zonas urbanas, tornando eminente a busca, tanto em espaços físicos, quanto em recursos para acolher, suprir as necessidades e garantir qualidade de vida para essas pessoas. As cidades que compartilham e implantam esse tipo de planejamento são denominadas de cidades sustentáveis.

Para definir cidades sustentáveis se faz necessário entender o conceito de sustentabilidade urbana. Em razão disto, Acselrad (1999) define como capacidade das políticas urbanas se adaptarem à oferta de serviços, à qualidade e à quantidade das demandas sociais, buscando $\mathrm{o}$ equilíbrio entre as diligências de serviços urbanos e investimentos em infraestrutura. Ainda é necessário adicionar o uso racional dos recursos humanos e a estruturação do ambiente urbano, baseado na interação com o clima e na supressão das necessidades urbanas, com um mínimo de transferência de dejetos para o meio ambiente.

Segundo Sampaio (2009), o debate sobre a sustentabilidade urbana surge da necessidade do entendimento sobre o conjunto de problemas relacionados à qualidade de vida urbana, tais como: alterações climáticas, esgotamento de energias fósseis e minerais, desmatamento desenfreado, violência, desigualdade social, transporte público escasso, entre outros. Considerando as definições apresentadas, pode-se definir uma cidade sustentável ou inteligente como um local onde ocorre um planejamento que permite implantar determinadas ações e políticas de desenvolvimento voltadas às práticas sustentáveis, de forma a respeitar e conservar, tanto o meio natural, quanto o meio urbano e a qualidade de vida nestes ambientes. Quando o planejamento é o mecanismo dominante, maior será o aumento da concentração populacional nas áreas urbanas $\mathrm{e}$ maior a exploração dos recursos naturais. A busca por uma melhora da qualidade de vida fez com que as ações humanas causassem muito mais danos ao meio ambiente.

As cidades sustentáveis ou inteligentes, conforme Kanter \& Litow (2009), são aquelas capazes de conectar de forma inovativa as infraestruturas físicas e de TIC (Tecnologias da Informação e Comunicação), de forma eficiente e eficaz, convergindo os aspectos organizacionais, normativos, sociais e tecnológicos a fim de melhorar as condições de sustentabilidade e de qualidade vida da população. Uma outra definição é a de Giffinger \& Gudrun (2010), caracterizandoas como aquelas que consideram uma visão de futuro em várias vertentes - economia, pessoas, governança, mobilidade, meio ambiente $\mathrm{e}$ qualidade de vida, e são construídas sobre a combinação inteligente de atitudes decisivas, independentes e conscientes dos atores que nelas atuam.

A partir das ações humanas relacionadas à ocupação de terra e utilização de recursos naturais ocorreu o aumento no interesse da implantação dessas cidades sustentáveis. Este interesse foi observado, tanto na esfera governamental, quanto de empresas que galgam, mesmo que em longo prazo, uma maior visibilidade e a valorização de seu empreendimento. Porém, para se estabelecer uma cidade sustentável, necessita-se do cumprimento de certos objetivos específicos, pois eles trarão benefícios, tanto para a cidade em si, quanto para o melhoramento da estrutura e da qualidade de vida da população (Rossetto, 2003).

Nessa perspectiva, observa-se em vários casos que mecanismos oriundos da tecnologia voltada ao planejamento das grandes cidades têm o 
poder de contribuir e beneficiar os efeitos de sua sustentabilidade. Logo, considerar a aplicação da modelagem BIM e CIM, seus pontos positivos e sua relevância no auxílio de projetos urbanos, faz deles influentes dispositivos que integram as cidades denominadas inteligentes, uma vez que, usando essas modelagens, o reflexo se dá no aumento da velocidade das interações, dando condições para o suprimento das necessidades básicas e promovendo o aumento da qualidade de vida como um todo.

Para Demantova \& Rutkowski (2007) não é possível preservar algo para o futuro, sem que exista envolvimento da sociedade atual, visto que esta é que se relaciona com o meio, ou melhor, com o espaço, na construção do futuro desejado. A situação perfeita é quando, tanto o governo, quanto a população sentem interesse na preservação do meio e na qualidade de vida, montando equipes com pensamento estratégico aguçado e qualidades técnicas elevadas, onde administram com sensibilidade as cidades utilizando softwares que abarcam as plataformas do SIG's, BIM e CIM. Segundo a lista da Economist Intelligence UnitEIU (2015), a cidade de Vancouver (Canadá), por exemplo, permaneceu no topo do ranking de cidade mais habitável do mundo, por diversos anos consecutivos. Esta posição favorável de Vancouver se deve aos mais de 200 parques dedicados às áreas verdes, aos programas de conscientização que influenciam os moradores a fazer práticas limpas para diminuir o impacto ambiental e aos incentivos fiscais para quem utiliza energia solar, o que faz com que cerca de $90 \%$ da energia utilizada na cidade venha de fontes renováveis, garantindo que Vancouver conte com a menor pegada de carbono da América do Norte, sendo uma das cidades mais sustentáveis do mundo (Toronto Sun, 2011).

No Brasil, um dos exemplos mais em evidência é a cidade de Curitiba, que chegou a receber, popularmente, a denominação de "Cidade Modelo" em razão de suas ações voltadas à preservação do meio e da qualidade de vida, bem como a infraestrutura oferecida para seus cidadãos (Bittencourt, 2006). A cidade conta com uma estimativa de $228.500 \mathrm{~m}^{2}$ de parques com áreas verdes e de lazer; presume-se que cerca de $70 \%$ da população da cidade se beneficie do transporte público eficiente. Além disso, a cidade é um nome de grande destaque quanto a baixa emissão de dióxido de carbono e geração de energia renovável. Considerando estes exemplos, mudanças na forma de como observar e gerir a cidade foram aplicadas e programas arrojados e banco de dados, com base nas conceituações de BIM, CIM e SIG's, foram utilizados. O monitoramento de áreas verdes, a mudança do setor viário tornando-o mais eficiente e os estudos de fontes renováveis refletem e comprovam a intrínseca necessidade de mão de obra e softwares sofisticados, o que faz o BIM e o CIM continuar crescente e ser uma tendência no planejamento das cidades inteligentes e/ou sustentáveis.

\section{Conclusão}

A modelagem oriunda do BIM e CIM e o seu vasto e integrado banco de dados pode proporcionar diversos benefícios para o ramo do design, engenharia, arquitetura e áreas afins, auxiliando na gestão de projetos. Observando a área disponível para construção e os recursos naturais da região, pode-se criar cidades seguindo o modelo sustentável, potencializando a geração de energia, uso da água e solo, transição/sazonalidade populacional e reduzindo custos desnecessários. Além disso, a possibilidade de urbanização e a arborização no próprio modelo corrobora as discussões sobre o microclima das cidades e sua relação com a sociedade.

\section{Agradecimentos}

Agradecemos à Fundação de Aparo a Ciência e Tecnologia de Pernambuco-FACEPE pelo incentivo dado à realização desta pesquisa. Ao Laboratório de Sensoriamento Remoto e Geoprocessamento-SERGEO pela colaboração na discussão dos resultados.

\section{Referências}

ACSELRAD, H. B. 1999. Discursos da sustentabilidade urbana. Estudos urbanos e regionais $\mathrm{n}^{\circ} 1$. Primeira edição.

ALLIANCE - Building Smart. 2009. Pennsylvania State University Releases BIM Project Execution Planning Guide [WWW] Building Smart Alliance. Disponível em: http://www.buildingsmartalliance.org/index.php/b sa/newsevents/news/Entry/BIMprojectexecutionpl anningguide. Acesso em: 11/11/2016.

AMORIM, A. L. 2015. Discutindo City Information Modeling (CIM) e conceitos correlatos. Gestão e Tecnologia de Projetos, v. 10, n. 2, p. 87-99.

ARANDA, M. G.; CRAWFORD, J.; CHEVES, A.; FROESE, T.2008. Building Information Modelling Demystified: Does it make business sense to adopt BIM. International Conference on Information Technology in Construction. Santiago. Chile.

BITTENCOURT, M. A. C. 2006. A informação e os indicadores de sustentabilidade: um estudo de 
caso no Observatório Regional Base de Indicadores de Sustentabilidade Metropolitano de Curitiba ORBIS MC - Universidade Federal de Santa Catarina, Centro Tecnológico. Programa de PósGraduação em Engenharia de Produção.

DAVE, B.; SCHMITT, G. 1994. Information systems for urban and design development. Environment and Planning B: Planning and Design, v. 21, p. 86-96.

DAVIS, K. 1977. Cidades: a urbanização da humanidade, ed. Zahar, Rio de Janeiro.

DAWOOD, N. N.; IQBAL, N. 2010. Building Information Modeling (BIM): A visual \& whole life cycle approach', CONVR2010, Sendai, Japan, November 4-5. In: MAKANAE, K.; YABUKI, N.; KASHIYAMA, K. (eds). Proceedings of the $10^{\text {th }}$ international conference on construction applications of virtual reality. CONVR2010 Organizing Committee, 2010, pp.7-14.

DDC - Departament of Design and Construction. 2012. BIM Guidelines. New York City, July. Disponível em: http://facilities.usc.edu/uploads/documents/cas/BI MGuidelines_VS1_6_2012.pdf. Acesso em: 13/11/2016.

DEMANTOVA, G. C.; RUTKOWSKI, E. W. 2007. A sustentabilidade urbana: simbiose necessária entre a sustentabilidade ambiental e a sustentabilidade social. Arquitextos-Periódico mensal de textos de arquitetura (Texto Especial 437). São Paulo: Portal Vitruvius.

DROGEMULLER, R. 2009. Can BIM be civil? Queensland Roads, v. 7, p. 47-55.

EASTMAN, C.; TEICHOLZ, P.; SACKS, R.; LISTON, K. 2008. BIM Handbook: A Guide to Building Information Modelling for Owners, Managers, Designers, Engineers, and Contractors. New Jersey. John Wiley \& Sons, Inc.

EIU - The Economist Intelligence Unit Limited. 2015. A Summary of the Liveability ranking and Overview. August 2015. Disponível em: http://www.vancouvereconomic.com/wpcontent/uploads/2015/08/EIU-Liveability-rankingAug-2015.pdf. Acesso em: 21 de fev. 2017.

GERBER, B. B.; RICE, S. 2010. The Perceived Value of Building Information Modelling in the U.S Building Industry. Journal of Information Technology in Construction, v. 15, n. 2, p.185-201.
GIFFINGER, R.; GUDRUN, H. 2010. Smarter Cities Ranking: An Effective Instrument for the Positioning of Cities? ACE: Architecture, City and Environment, v. 12, p. 7-25.

GIL, A. C. 2008. Métodos e técnicas de pesquisa social. 6. ed., São Paulo: Atlas. 220p.

GIL, J.; ALMEIDA, J.; DUARTE, J. 2011. The backbone of a City Information Model (CIM): Implementing a spatial data model for urban design. In: Education and Research Design in Europe, 29. Ljubljana. Proceedings... Ljubljana, University of Ljubljana / Faculty of Architecture.

HAMIL, S. 2013. The Potential of BIM to Reduce Waste During Design and Construction. Retrieved November 11, 2015. Disponível em: http://constructioncode.blogspot.co.uk/2013/03/th e-potential-of-BIM-to-reduce-waste.html. Acesso em: 13/11/2016.

IBGE, Censo Demográfico. 2010. Mapas; Mapas estatísticos; População-Brasil. Disponível em: https://cidades.ibge.gov.br/. Acesso em: setembro 10, 2016.

KANTER, R. M.; LITOW, S. S. 2009. Informed and interconnected: A manifesto for smarter cities. Harvard Business School General Management Unit Working Paper 09-141. Disponível em: http://papers.ssrn.com/sol3/papers.cfm?abstract_i $\mathrm{d}=1420236$. Acesso em: 14/08/2012.

KHEMLANI, L. 2013. Around the World with BIM. Analysis, Research and Review of AEC Technology. Retrieved 19 November.

MAGUIRE, D. J 2003. Improving CAD-GIS interoperability. ESRI, Winter.

OSMANI, M.; GLASS, J.; PRICE, A. D. F. 2006. Architect and Contractor Attitudes to Waste Minimisation. Waste and Resource Management, v. 159, n. 2 , p. $65-72$.

OYEDELE, L. O.; REGAN, M.; MEDING, J.; AHMED, A.; EBOHON, O. J.; ELNOKALY, A. 2013. Reducing Waste to Landfill in the UK: Identifying Impediments and Critical Solutions. World Journal of Science, Technology and Sustainable Development, v. 10, n. 2, p.1-4.

PPGAU - Programa de Pós-Graduação em Arquitetura e Urbanismo. 2015. Encontro Nacional de Tecnologia de Informação e Comunicação na Construção. Disponível em: 
http://prpg.usp.br/PPGAU/eventos/mostrar/5432. Acesso em: 10/11/2015.

REZGUI, Y.; BEACH, T.; RANA, O. 2013. A Governance Approach for BIM Management Across Lifecycle and Supply Chains Using MixedModes of Information Delivery. Journal of Civil Engineering and Management, v. 19, n. 2, p. 239258.

ROSSETTO, A. M. 2003. Proposta de um sistema integrado de gestão do ambiente urbano (SIGAU), para o desenvolvimento sustentável de cidades. Programa de Pós-Graduação em Engenharia de Produção (PPGEP) da Universidade Federal de Santa Catarina (UFSC), Florianópolis 2003.

SAH, V.; CORY, C. 2009. Building Information Modelling: An Academic Perspective. Technology Interface Journal, Winter Special Issue, v. 10, n. 2, p. $01-11$.

SAMPAIO, D. T. 2009. Sustentabilidade urbana: conceitos e controvérsias. 5 Encontro Nacional e III Latino-americano sobre edificações e comunidades sustentáveis 2009.

SANTOS, M. 1994. Técnica, espaço, tempo: globalização e meio técnico-científico informacional. São Paulo: Hucitec.

SPOSITO, M. E. B. 2004. O chão em pedaços: urbanização, economia e cidades no estado de São Paulo. Presidente Prudente: [s.n.].
STADLER, A.; KOLBE, T. H. 2007. Spatiosemantic coherence in the integration of 3D city models. Proceedings of the 5th International Symposium on Spatial Data Quality, Enschede.

STOJANOVSKI, T. 2013. City Information Modeling (CIM) and Urbanism: blocks, connections, territories, people and situations. In: Symposium on Simulation for Architecture and Urban Design, San Diego. Disponível em: http://dl.acm. org/citation.cfm?id=2500016. Acesso em: 11 nov. 2015.

TORONTO SUN. 2011. Vancouver no longer the world's livable city: Survey. Disponível em: http://www.torontosun.com/2011/08/30/vancouve r-no-longer-the-worlds-most-liveable-city-survey. Acesso: 21 de fevereiro. 2017.

WATSON, A. 2010. BIM - a driver for change. Paper presented at the Proceedings of the. International Conference on Computing in Civil and Building Engineering, Leeds, UK

WEBSTER, C. 1993. GIS and the scientific inputs to urban planning. Part 1: description. Environment and Planning B: Planning and Design, v. 20, p. 709-728.

XU, X.; DING, L.; LUO, H.; MA, L. 2014. From Building Information Modeling to City Information Modeling. Journal of Information Technology in Construction, v. 19, p. 01-16. 